\title{
Disseminated Coinfection by Mycobacterium fortuitum and Talaromyces marneffei in a Non-HIV Case
}

\author{
Hongchun Huang',* \\ Jingmin Deng ${ }^{2, *}$ \\ Caixia Qin I I \\ Jianpeng Zhou (D) ${ }^{3}$ \\ Minchao Duan (iD) 4 \\ 'Department of Respiratory and Critical \\ Care Medicine, Guangxi Autonomous \\ Regional Jiangbin Hospital, Nanning, \\ Guangxi, 53002I, People's Republic of \\ China; ${ }^{2}$ Department of Respiratory and \\ Critical Care Medicine, The First \\ Affiliated Hospital of Guangxi Medical \\ University, Nanning, Guangxi, 53002I, \\ People's Republic of China; ${ }^{3}$ Department \\ of Respiratory and Critical Care \\ Medicine, The First People's Hospital of \\ Nanning, Nanning, Guangxi, 530022, \\ People's Republic of China; ${ }^{4}$ Department \\ of Respiratory and Critical Care \\ Medicine, Guangxi Medical University \\ Wuming Affiliated Hospital, Nanning, \\ Guangxi, 530199, People's Republic of \\ China
}

*These authors contributed equally to this work
Background: Mycobacterium fortuitum is a rapidly growing non-tuberculous mycobacterium (NTM) with weak pathogenicity. Here, we present a rare case of disseminated M. fortuitum and Talaromyces marneffei coinfection in a human immunodeficiency virus (HIV) negative patient.

Case Presentation: A 28-year-old female was admitted to our hospital due to 2 months of swelling of lymph nodes on the right side of her cervix, accompanied by repeated low fever for more than 1 month. Biopsy of the right cervical lymph node and endobronchial ultrasound-guided transbronchial fine needle aspiration (EBUS-TBNA) both suggested granulomatous inflammation. The bacterial culture and mycobacteria examination of the lesion as well as HIV antibody test were all negative. Disseminated T. marneffei infection was diagnosed by the quantitative polymerase chain reaction (qPCR) results from the blood showing 1798 copies/ul. In the meantime, treatment with amphotericin B combined with cefoxitin was administered for suspected NTM infection. However, the once-dropped fever recurred and the lymph nodes continued to swell. Metagenomics next-generation sequencing (mNGS) detection of the lymph nodes indicated M. fortuitum. After combination treatment with amphotericin B, voriconazole, linazolamide, and imipenem, the patient's body temperature returned to normal, the lymph node swelling was gradually reduced, and the lung lesion was absorbed.

Conclusion: We report the first case of an HIV-negative patient diagnosed with disseminated $M$. fortuitum and T. marneffei coinfection with nonspecific clinical manifestation, in order to heighten awareness of these infections.

Keywords: Mycobacterium fortuitum, Talaromyces marneffei, coinfection, non-HIV

\section{Introduction}

Mycobacterium fortuitum is widely distributed in natural environments with strong survivability $^{1}$ and can cause cutaneous and pulmonary infection, lymphadenitis, and disseminated disease. M. fortuitum pulmonary disease is associated with multiple conditions, such as gastroesophageal disease ${ }^{2}$ and structural pulmonary disorders, while disseminated disease is usually associated with immunodeficiency disorders. ${ }^{3}$ Pathogenesis of NTM remains unclear and potential pathogenicity factors include hydrophobic outer membrane rich in lipids, surface adhesive ability, biofilm and aerosol formation. ${ }^{4}$ Macrophages are critical in the host's immune response to NTM. Specifically, the pathogen-recognition receptors of macrophages recognize pathogen-associated molecular patterns on the NTM.
Correspondence: Minchao Duan Department of Respiratory and Critical Care Medicine, Guangxi Medical University Wuming Affiliated Hospital, 26 Yongning Road, Wuming District, Nanning, Guangxi, 530199, People's Republic of China

$\mathrm{Tel} / \mathrm{Fax}+86$ I55077 I I 966

Email fortuitum@sohu.com 
The macrophage then phagocytoses the NTM to form a phagosome, which fuses with the lysosome to form a phagolysosome to eliminate NTM. Macrophages act as antigen-presenting cells to trigger downstream activation of cellular and humoral adaptive immune responses. Macrophages can also kill NTM by producing antimicrobial active substances such as reactive oxygen species and reactive nitrogen species or by autophagy/necrosis mechanisms. NTM can evade the attack of the immune system by impairing phagosome maturation and autophagy/necrosis and promoting chronic infection. ${ }^{5}$ There is some evidence that NTM uses apoptotic macrophages to facilitate the spreading and survival in the host. ${ }^{6}$ Besides, in response to stimuli from NTM pathogens, the macrophage lineage expresses polarized functional properties, namely activated macrophages (M1) and activated macrophages (M2), with M2 polarization regulating immunity and preventing NTM injury during the advanced stages of infection, which is a critical mechanism of intracellular infection. ${ }^{7,8}$ Moreover, granuloma is also a sanctuary for NTM. In a zebrafish model, $M$. fortuitum can invade macrophages to develop mycobacterial granuloma, leading to chronic infection. ${ }^{9}$

As an intracellular pathogen, T. marneffei also inhibits phagosome maturation. ${ }^{10}$ T. marneffei can also withstand host-cell defenses such as antioxidants, heat shock proteins, and well-conserved mitogen-activated protein kinases signaling cascades. ${ }^{11}$ T. marneffei is capable of driving macrophage polarization, ${ }^{12}$ but it remains unclear if T. marneffei can induce macrophages to M2 polarization in order to survive in the host during infection.

Clinically, M.f ortuitum usually causes colonized or transient infection in respiratory tracts. ${ }^{13,14}$ Coinfection by $M$. fortuitum and another intracellular pathogen has been reported in patients with immunodeficiency such as acquired immune deficiency syndrome but not in healthy individuals. ${ }^{15,16}$ Coinfections of $T$. marneffei with other NTM have also been reported, ${ }^{17-21}$ but not with M. fortuitum. M. fortuitum and T. marneffei are opportunistic pathogens, with the majority of cases of disseminated infection found in patients with immunodeficiency diseases. ${ }^{22-24}$ In our case, a non-HIV patient had coinfection with two pathogens, and we attempted to rule out potential primary immunodeficiency.

Here, we report the first case of disseminated M. fortuitum and T. marneffei coinfection in an HIVnegative patient with no specific clinical symptoms and a difficult early diagnosis, to provide clinical experience.

\section{Case Presentation}

A 28-year-old female was admitted to our hospital on August 17, 2017, with lymphadenomegaly on her right cervical area for approximately 2 months and repeated fever for more than 1 month, accompanied by a red rash, edema, and painful subcutaneous nodules in bilateral legs. Physical examination revealed lymphadenopathy in the right cervical bone and painful erythema nodosum in the bilateral calf. The patient had a history of pet rat bites in 2015.

Laboratory tests showed that hemoglobin $(9.4 \mathrm{~g} / \mathrm{dL})$ and hematocrit $(28.8 \%)$ levels were decreased, while white blood cell count $(16.27 \times 109 / \mathrm{L})$, neutrophils $(78.6 \%)$, platelet count $(418 \times 109 / \mathrm{L})$, blood C-reactive protein $(138.15 \mathrm{mg} / \mathrm{L})$ and erythrocyte sedimentation rate $(88 \mathrm{~mm} / \mathrm{h})$ were elevated. HIV serology and serum cryptococcal capsular antigen tests were negative. Immunoglobulin levels and lymphocyte subsets were normal. T cell spot test (T-SPOT) was indeterminate. Culture of blood, sputum, bronchoalveolar lavage fluid (BALF), and skin lesions were all negative. Chest computed tomography (CT) displayed patchy infiltrates in the right upper lung with enlargement of hilus pulmonis and mediastinal lymph nodes (Figure 1A). Pathological examination of the cervical lymph nodes suggested granulomatous inflammation, but acid-fast staining and periodic acid-Schiff (PAS) staining were negative (Figure 2A and B). EBUS-TBNA biopsy examination showed scattered granulomatous inflammation. During the course, empirical treatments with cefoxitin (CFX), levofloxacin (LVFX), moxifloxacin (MFX), cefodizime (CDZ), clarithromycin (CLA), etimicin (ETM) and anti-tuberculosis isoniazid $(\mathrm{H})$, rifampicin $(\mathrm{R})$, pyrazinamide $(\mathrm{Z})$, ethambutol (E) regimen were successively administered, but no improvement was observed. By the end of October 2017, the patient developed pain in the head, right shoulder, gingiva, and kneejoint. Cerebrospinal fluid examination was normal, its smear and culture were all negative. Although bone marrow biopsy was consistent with infection and there was no evidence of hematological malignancy, the culture of bone marrow and lymph node tissue was both negative. Pathology examination of cervical lymph nodes showed suppurative necrotizing lymphadenitis, no fungus, and PAS (-). Positron emission tomography-computed tomography displayed enlargement of multiple lymph nodes of the right hilar, mediastinum, right supraclavicular and right neck, splenomegaly and increased bone metabolism in multiple places. The symptoms persisted after multiple rounds of antibiotic treatments, raising the possibility of a fungal infection. As the patient had a history of rat bites, an important natural reservoir for T. marneffei, we 
A

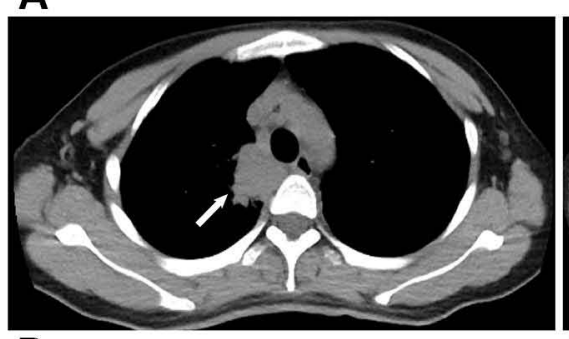

D

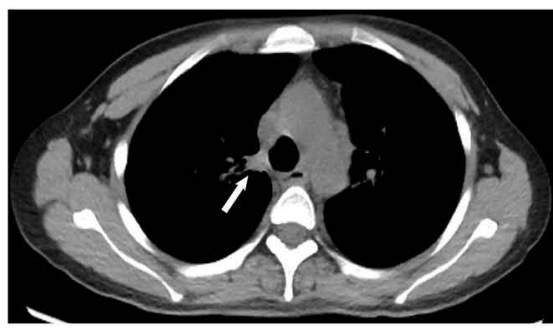

B

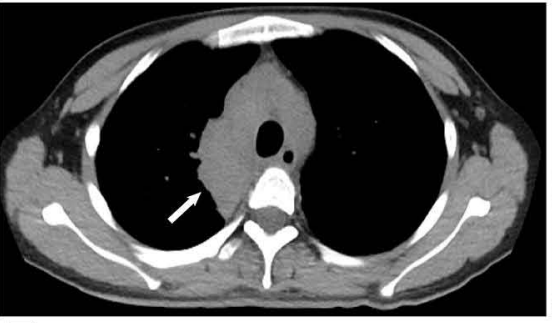

E

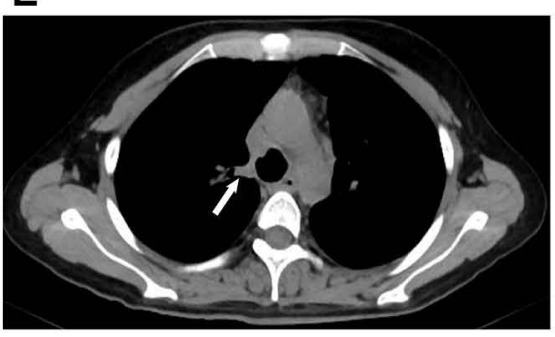

C

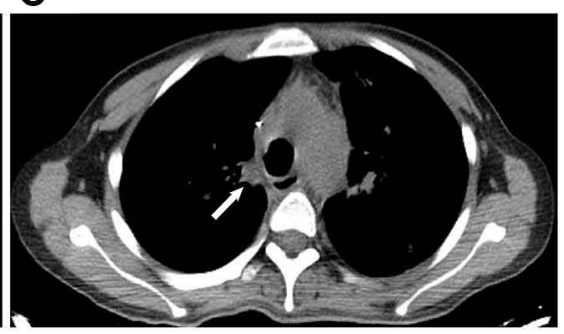

Figure I (A and B) shows lymph node enlargement in the hilus pulmonis zone; (B-E) shows that enlarged lymph nodes shrank gradually in the hilus pulmonis zone.

A

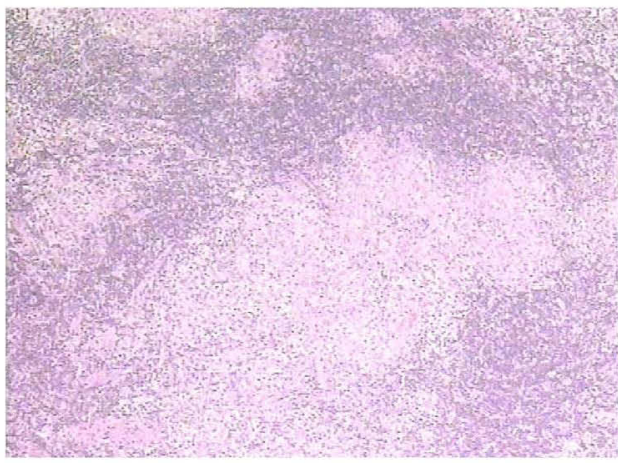

B

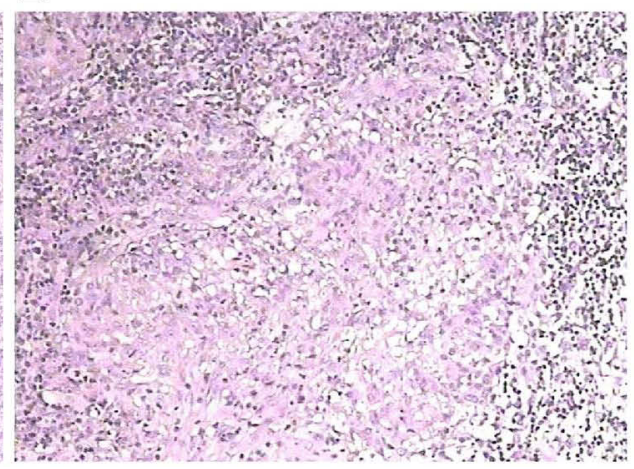

Figure 2 (A and B) pathological examination of H\&E staining (H\&E, 100x; H\&E, 400x) from the cervical lymph nodes suggested granulomatous inflammation. Granulomas is composed of epithelioid cells in the lymph nodes. PAS(-).

conducted T. marneffei qPCR and obtained a result of 1798 copies/uL. Therefore, the patient was diagnosed with disseminated T. marneffei infection, and intravenous antifungal therapy with amphotericin B liposome (AMPB) commenced on November 6, 2017. Owing to suspicion of coinfection with NTM, the patient was also treated with cefoxitin beginning on November 10, 2017. Subsequently, her body temperature dropped to normal, and symptoms of cough and sputum improved. The patient returned to the local hospital and continued treatment with itraconazole and cefoxitin.

However, the lymph nodes remained enlarged. In December 2018, the patient presented with hemoptysis and recurrent intermittent fever, cough, sputum, fever, and rash. The patient presented with recurrent fever, rash and lymph node enlargement, which did not improve despite treatment with various antibiotics and anti-infective treatment for
T. marneffei and M. tuberculosis. The pathological manifestations were granulomatous inflammation, but we could not rule out NTM infection because the cultures for M. tuberculosis and NTM were negative. Therefore, we performed an mNGS test, which revealed $M$. fortuitum in the lymph nodes. Additionally, in January 2020, qPCR analysis of a BALF sample detected T. marneffei. The patient was diagnosed with coinfection with M. fortuitum and T. marneffei and was treated with a combination of amphotericin B, voriconazole (VRC), linezolid (LZD) and ETM. Her body temperature returned to normal and respiratory symptoms improved. On March 19, 2020, chest CT showed patchy infiltrates, banded and nodular shadows, as well as pleural effusion (Figure 3A). Emission computed tomography suggested abnormal concentration of imaging agent in various parts of the bone. One month later, the therapy was adjusted to a combination regimen of VRC, LZD, 
A

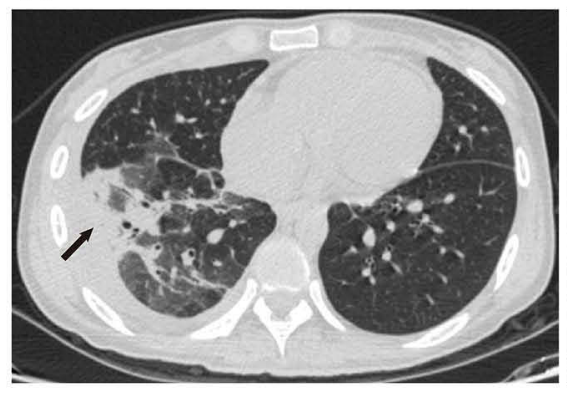

B

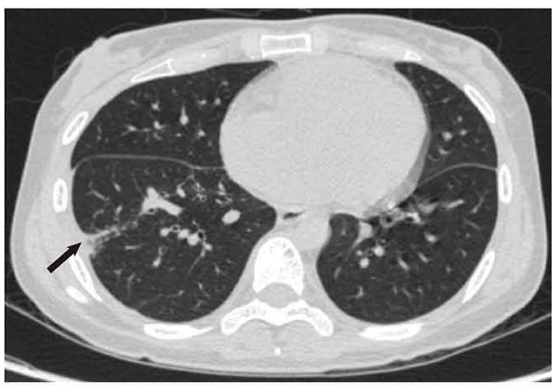

C

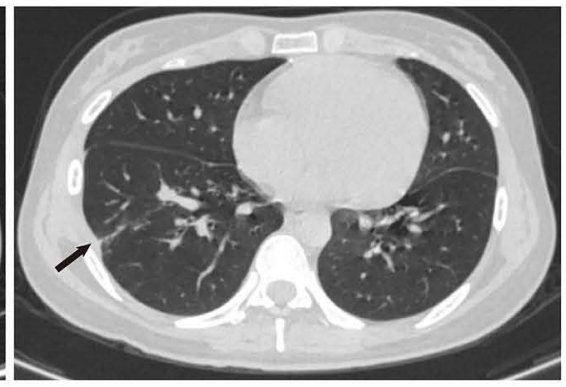

Figure 3 (A) Chest CT shows patchy infiltrates, banded and nodular shadows, pleural effusion. (A-C) Lesions in the lower lobe of the right lung and the pleural effusion absorbed gradually.

MFX and CLA, which have continued to date. During followup in May and August 2020, the patient had no fever, the symptoms of cough and sputum had improved, and the rash had subsided. Re-examination of chest $\mathrm{CT}$ indicated that the enlarged hilar lymph nodes had gradually shrank (Figure 1BE), and the lung lesions were significantly absorbed (Figure 3A-C). The patient experienced significant pain for more than 2 years between onset to the definitive diagnosis. The hospitalization and drug administration process throughout the history is summarized in Figure 4. Unfortunately, we overlooked the possibility of immunodeficiency in HIVnegative patient coinfected with M. fortuitum and T. marneffei.

\section{Discussion and Conclusions}

The diagnosis of disseminated NTM infection was mainly based on positive blood culture, followed by positive culture of bone marrow, liver, and lymph node tissue. ${ }^{25}$
In this case, we detected M. fortuitum in the lymph node samples, which is the best evidence of tissue infection, rather than colonization. Meanwhile, we made the diagnosis of disseminated T. marneffei by the detection of high copies of $T$. marneffei with $\mathrm{qPCR}^{26}$ The patient was finally diagnosed with disseminated M. fortuitum and T. marneffei infection.

We noticed that the patient had recurrent skin rashes throughout the disease. Either M. fortuitum infection or T. marneffei infection has cutaneous manifestation. Clinical signs of $M$. fortuitum infection are abscess, nodule, plaque, and papule. The most commonly involved sites are the legs. ${ }^{27}$ This is similar to our case of painful erythema nodosum in the bilateral legs. While for T. marneffei infection, characteristic skin manifestations are umbilicated papules. ${ }^{28}$ The skin lesions are mainly distributed on the face, scalp, upper extremities, and

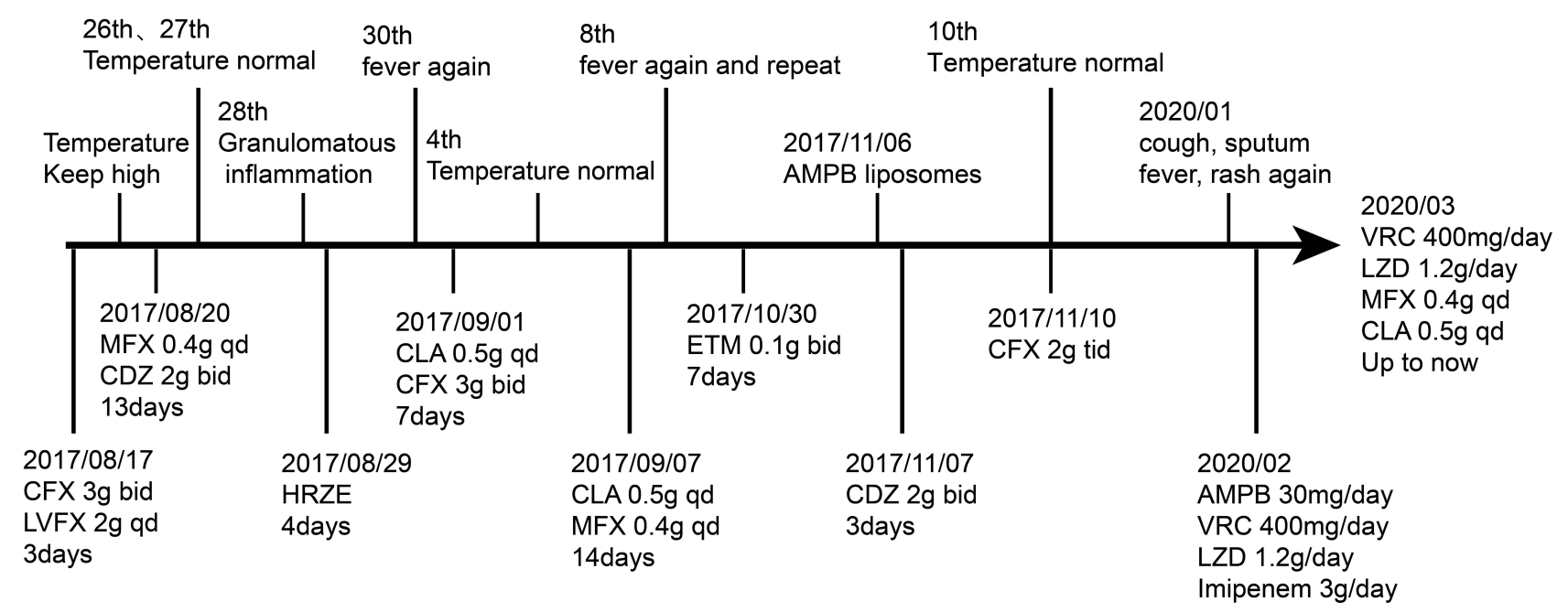

Figure 4 Treatment and manifestation timeline.

Abbreviations: CFX, cefoxitin; LVFX, levofloxacin; MFX, moxifloxacin; CDZ, cefodizime; CLA, clarithromycin; ETM, etimicin; H, isoniazid; R, rifampicin; Z, pyrazinamide; E, ethambutol; AMPB, amphotericin B liposomes; VRC, voriconazole; LZD, linezolid. 
trunk. Immune deficiency and trauma history are risk factors for NTM skin infections. ${ }^{29}$ In the case of M. fortuitum, patients with single skin lesions are almost always immunocompetent; however, multiple lesions are usually in the context of immunosuppression, and related to disseminated disease. ${ }^{30}$ In our case, the patient had a history of trauma, but clinical morphology showed multiple lesions. Combined with coinfection of two opportunistic pathogens, $M$. fortuitum and T. marneffei, the patient was considered to have an immunodeficiency disease. We tried to find evidence of immunodeficiency but failed to do so. The young female was HIV-negative, without a history of corticosteroids or immunosuppressive medicine, and no underlying diseases such as structural pulmonary disease, myeloma, autoimmune diseases, hematological malignancies, solid tumors, or diabetes. Her lymphocyte and immunoglobulin levels were also normal. However, with the data provided, we cannot exclude that the patient has an underlying primary immunodeficiency.

There is no specific treatment for M. fortuitum infection. It was recently found that rapidly growing mycobacterium harbors mutations in the erythromycin methylase (erm) gene, resulting in macrolide resistance. ${ }^{31}$ Macrolides work by binding to the $50 \mathrm{~S}$ ribosomal subunit and inhibiting peptide synthesis. The erm mutant can inhibit binding to ribosomes and reduce the antimicrobial activity of macrolides. Recent guidelines ${ }^{32}$ recommend treating M. abscess pulmonary infection according to the presence or absence of the erm mutation. For non-mutant strains, initial treatment should consist of three active drugs including macrolides. For mutant strains, initial treatment should consist of four active drugs including macrolides, which is useful due to its immunomodulatory properties. M. fortuitum as a rapidly growing mycobacterium can be treated with the regimen used for M. abscess. Recently, an in vitro sensitivity test in Shanghai ${ }^{33}$ confirmed that $M$. fortuitum was resistant to macrolides, and the resistance rates to clarithromycin, azithromycin, cefoxitin, and moxifloxacin were $94 \%, 100 \%, 88 \%$, and $59 \%$, respectively, while it showed good sensitivity to linezolid and imipenem. In our case, the initial therapy of cefoxitin, moxifloxacin, clarithromycin, and levofloxacin was ineffective. The patient's condition improved after administration of the combination of linezolid, moxifloxacin, clarithromycin and imipenem. However, patients with IFN- $\gamma$ autoimmune disease may have recurrent infections. Combination with rituximab can reduce the risk of recurrence. The patient in this study has not experienced disease recurrence to date and is undergoing regular follow-ups.

In summary, M. fortuitum is usually considered to have low virulence and causes colonized or transient infection rather than pathopoiesis in the respiratory tract. We report the first case of coinfection with $M$. fortuitum and T. marneffei in an HIV-negative patient. This finding serves as a reminder to clinicians that $M$. fortuitum infection may occur in a seemingly healthy patient, and that patients with similar disseminated infections should be evaluated for primary immunodeficiency.

\section{Abbreviations}

NTM, non-tuberculous mycobacteriosis; HIV, human immunodeficiency virus; EBUS-TBNA, endobronchial ultrasound-transbronchial lung needle aspiration; qPCR, quantitative polymerase chain reaction; mNGS, metagenomics next-generation sequencing; T-SPOT, $\mathrm{T}$ cell spot test; BALF, bronchoalveolar lavage fluid; CT, computed tomography; PAS, periodic acid-Schiff; CFX, cefoxitin; LVFX, levofloxacin; MFX, moxifloxacin; CDZ, cefodizime; CLA, clarithromycin; ETM, etimicin; $\mathrm{H}$, isoniazid; $\mathrm{R}$, rifampicin; $\mathrm{Z}$, pyrazinamide; $\mathrm{E}$, ethambutol; $\mathrm{AMPB}$, amphotericin B liposomes; VRC, voriconazole; LZD, linezolid; erm, erythromycin methylase.

\section{Ethics Approval and Informed Consent}

Written informed consent to have the case details and any accompanying images published has been provided by the patient. No ethical committee approval was required for this study as the data were analyzed in a retrospective manner.

\section{Acknowledgments}

All authors thank the patient and her families for their support.

\section{Author Contributions}

All authors made substantial contributions to the conception and design, acquisition of data, or analysis and interpretation of data; participated in drafting the article or revising it substantially, agreed to submit to the current journal, gave final approval of the version to be published; and agreed to take responsibility for all aspects of the work. 


\section{Funding}

There is no funding to report.

\section{Disclosure}

The authors report no conflicts of interest in this work.

\section{References}

1. Zhuang B, Wu C, Lin G. [Resistance of Mycobacterium fortuitum against varied physical factors and its survival in different surroundings]. Zhonghua Jie He He Hu Xi Za Zhi. 2001;24 (11):679-681. Chinese.

2. Okamori S, Asakura T, Nishimura $T$, et al. Natural history of Mycobacterium fortuitum pulmonary infection presenting with migratory infiltrates: a case report with microbiological analysis. BMC Infect Dis. 2018;18(1):1. doi:10.1186/s12879-017-2892-9

3. Corti M, Soto I, Villafañe F, Esquivel P, Di Lonardo M. [Disseminated infection due to Mycobacterium fortuitum in an AIDS patient]. Medicina (B Aires). 1999;59(3):274-276. Spanish.

4. To K, Cao R, Yegiazaryan A, Owens J, Venketaraman V. General Overview of Nontuberculous Mycobacteria Opportunistic Pathogens: mycobacterium avium and Mycobacterium abscessus. J Clin Med. 2020;9(8):2541. doi:10.3390/jcm9082541

5. Shamaei M, Mirsaeidi M. Nontuberculous Mycobacteria, Macrophages, and Host Innate Immune Response. Infect Immun. 2021;89(8):e0081220. doi:10.1128/IAI.00812-20

6. Early J, Fischer K, Bermudez LE. Mycobacterium avium uses apoptotic macrophages as tools for spreading. Microb Pathog. 2011;50 (2):132-139. doi:10.1016/j.micpath.2010.12.004

7. Tomioka H, Tatano Y, Maw WW, Sano C, Kanehiro Y, Shimizu T. Characteristics of suppressor macrophages induced by mycobacterial and protozoal infections in relation to alternatively activated M2 macrophages. Clin Dev Immunol. 2012;2012:635451. doi:10.1155/ 2012/635451

8. Thiriot JD, Martinez-Martinez YB, Endsley JJ, Torres AG. Hacking the host: exploitation of macrophage polarization by intracellular bacterial pathogens. Pathog Dis. 2020;78(1):ftaa009. doi:10.1093/ femspd/ftaa009

9. Johansen MD, Kremer L. CFTR Depletion Confers Hypersusceptibility to Mycobacterium fortuitum in a Zebrafish Model. Front Cell Infect Microbiol. 2020;10:357. doi:10.3389/ fcimb.2020.00357

10. Lu S, Hu Y, Lu C, Zhang J, Li X, Xi L. Development of in vitro macrophage system to evaluate phagocytosis and intracellular fate of Penicillium marneffei conidia. Mycopathologia. 2013;176(12):11-22. doi:10.1007/s11046-013-9650-3

11. Pongpom M, Vanittanakom $\mathrm{P}$, Nimmanee $\mathrm{P}$, Cooper CR Jr, Vanittanakom N. Adaptation to macrophage killing by Talaromyces marneffei. Future Sci OA. 2017;3(3):FSO215. doi:10.4155/fsoa2017-0032

12. Lu S, Li D, Xi L, Calderone R. Interplay of interferon-gamma and macrophage polarization during Talaromyces marneffei infection. Microb Pathog. 2019;134:103594. doi:10.1016/j.micpath.20 19.103594

13. Duan H, Han X, Wang Q, et al. Clinical Significance of Nontuberculous Mycobacteria Isolated From Respiratory Specimens in a Chinese Tuberculosis Tertiary Care Center. Sci Rep. 2016;6 (1):36299. doi:10.1038/srep36299

14. Park S, Suh GY, Chung MP, et al. Clinical significance of Mycobacterium fortuitum isolated from respiratory specimens. Respir Med. 2008;102(3):437-442. doi:10.1016/j.rmed.2007.10.005
15. Hirabayashi R, Nakagawa A, Takegawa H, Tomii K. A case of pleural effusion caused by Mycobacterium fortuitum and Mycobacterium mageritense coinfection. BMC Infect Dis. 2019;19 (1):720. doi:10.1186/s12879-019-4366-8

16. Soto-Arquínigo L, García-Pareja M, Gotuzzo-Herencia E, LeguaLeiva P, Sánchez-Herrera M. [Co-infection by Mycobacterium fortuitum and Mycobacterium tuberculosis in splenic abscesses in a patient with HIV]. Rev Peru Med Exp Salud Publica. 2017;34 (2):328-331. doi:10.17843/rpmesp.2017.342.2470. Catalan.

17. Su -S-S, Zhang S-N, Ye J-R, et al. $<\mathrm{p}>$ Disseminated Talaromyces marneffei And Mycobacterium avium Infection Accompanied Sweet's Syndrome In A Patient With Anti-Interferon- $\gamma$ Autoantibodies: a Case Report. Infect Drug Resist. 2019;12:3189-3195. doi:10.2147/IDR.S218836

18. Xu H, Liu D, He X, Zheng D, Deng Y. Sweet's Syndrome Associated with Talaromyces marneffei and Mycobacterium abscessus Infection Due to Anti-interferon-gamma Autoantibodies. Indian J Dermatol. 2018;63(5):428-430. doi:10.4103/ijd.IJD_362_17

19. Pruetpongpun N, Khawcharoenporn $T$, Damronglerd $P$, et al. Disseminated Talaromyces marneffei and Mycobacterium abscessus in a Patient With Anti-Interferon- $\gamma$ Autoantibodies. Open Forum Infect Dis. 2016;3(2):ofw093. doi:10.1093/ofid/ofw093

20. Seok H, Ko JH, Shin I, et al. Disseminated Talaromyces marneffei and Mycobacterium intracellulare coinfection in an HIV-infected patient. Int J Infect Dis. 2015;38:86-88. doi:10.1016/j.ijid.2015.07.020

21. Zhang J, Huang X, Zhang X, et al. Coinfection of disseminated Talaromyces marneffei and Mycobacteria kansasii in a patient with papillary thyroid cancer: a case report. Medicine (Baltimore). 2017;96(52):e9072. doi:10.1097/MD.0000000000009072

22. Ding X, Huang H, Zhong L, et al. Disseminated Talaromyces marneffei Infection in a Non-HIV Infant With a Homozygous Private Variant of RELB. Front Cell Infect Microbiol. 2021;11:605589. doi:10.3389/fcimb.2021.605589

23. Chetchotisakd P, Kiertiburanakul S, Mootsikapun P, Assanasen S, Chaiwarith R, Anunnatsiri S. Disseminated nontuberculous mycobacterial infection in patients who are not infected with HIV in Thailand. Clin Infect Dis. 2007;45(4):421-427. doi:10.1086/520030

24. Santos PE, Piontelli E, Shea YR, et al. Penicillium piceum infection: diagnosis and successful treatment in chronic granulomatous disease. Med Mycol. 2006;44(8):749-753. doi:10.1080/13693780600967089

25. Davidson PT. The other mycobacteria. Chest. 1979;75(2):110-111. doi:10.1378/chest.75.2.110

26. Hien H, Thanh TT, Thu N, et al. Development and evaluation of a real-time polymerase chain reaction assay for the rapid detection of Talaromyces marneffei MP1 gene in human plasma. Mycoses. 2016;59(12):773-780. doi:10.1111/myc. 12530

27. Chirasuthat P, Triyangkulsri K, Rutnin S, Chanprapaph K, Vachiramon V. Cutaneous nontuberculous mycobacterial infection in Thailand: a 7-year retrospective review. Medicine (Baltimore). 2020;99(10):e19355. doi:10.1097/MD.0000000000019355

28. Ungpakorn R. Cutaneous manifestations of Penicillium marneffei infection. Curr Opin Infect Dis. 2000;13(2):129-134. doi:10.1097/ 00001432-200004000-00006

29. Lee WJ, Kang SM, Sung H, et al. Non-tuberculous mycobacterial infections of the skin: a retrospective study of 29 cases. J Dermatol. 2010;37(11):965-972. doi:10.1111/j.1346-8138.2010.00960.x

30. Kothavade RJ, Dhurat RS, Mishra SN, Kothavade UR. Clinical and laboratory aspects of the diagnosis and management of cutaneous and subcutaneous infections caused by rapidly growing mycobacteria. Eur J Clin Microbiol Infect Dis. 2013;32(2):161-188. doi:10.1007/s10096012-1766-8

31. Nash KA, Zhang Y, Brown-Elliott BA, Wallace RJ Jr. Molecular basis of intrinsic macrolide resistance in clinical isolates of Mycobacterium fortuitum. $J$ Antimicrob Chemother. 2005;55 (2):170-177. doi:10.1093/jac/dkh523 
32. Daley CL, Iaccarino JM, Lange C, et al. Treatment of Nontuberculous Mycobacterial Pulmonary Disease: an Official ATS/ ERS/ESCMID/IDSA Clinical Practice Guideline: executive Summary. Clin Infect Dis. 2020;71(4):e1-e36. doi:10.1093/cid/ ciaa 241
33. Shen Y, Wang X, Jin J, et al. In Vitro Susceptibility of Mycobacterium abscessus and Mycobacterium fortuitum Isolates to 30 Antibiotics. Biomed Res Int. 2018;2018:4902941. doi:10.1155/ 2018/4902941

\section{Publish your work in this journal}

Infection and Drug Resistance is an international, peer-reviewed openaccess journal that focuses on the optimal treatment of infection (bacterial, fungal and viral) and the development and institution of preventive strategies to minimize the development and spread of resistance. The journal is specifically concerned with the epidemiology of

Submit your manuscript here: https://www.dovepress.com/infection-and-drug-resistance-journa antibiotic resistance and the mechanisms of resistance development and diffusion in both hospitals and the community. The manuscript management system is completely online and includes a very quick and fair peerreview system, which is all easy to use. Visit http://www.dovepress.com/ testimonials.php to read real quotes from published authors. 